\title{
Predictors of Regional Lymph Node Recurrence after Initial Thyroidectomy in Patients with Thyroid Cancer
}

\author{
Amirsina Sharifi, Abolfazl Shojaeifard, Ahmadreza Soroush, Mehdi Jafari, Ali Ghorbani \\ Abdehgah, and Hossein Mahmoudzade
}

Department of General Surgery, Shariati Hospital, North Kargar Street, Tehran, Iran

Correspondence should be addressed to Abolfazl Shojaeifard; fard.sh@gmail.com

Received 27 December 2015; Accepted 10 April 2016

Academic Editor: Gary L. Francis

Copyright (c) 2016 Amirsina Sharifi et al. This is an open access article distributed under the Creative Commons Attribution License, which permits unrestricted use, distribution, and reproduction in any medium, provided the original work is properly cited.

Background. Regional lymph node recurrence (RLNR) is common in patients with thyroid cancer but clinicopathological predictors are unclear. We aimed to clarify these predictors and identify patients who would benefit from prophylactic lymph node dissection the most. Method. 343 patients with different types of thyroid cancer were analyzed retrospectively. All patients underwent total thyroidectomy between 2007 and 2013. Results. The median \pm interquartile range of patients' age was $40 \pm 25$ years. 245 (71.4\%) patients were female. Regarding the risk of regional lymph node recurrence, we found that male gender, age $\geq 45$ years, non-PTC (i.e., medullary, follicular, and anaplastic types) histopathology, T3 (i.e., tumor size $>4 \mathrm{~cm}$ in the greatest dimension limited to the thyroid or any tumor with minimal extrathyroid extension), stage IVa, and isolated cervical lymphadenopathy as initial manifestation (ICL) are significant risk factors. T3 $(p<0.001$; odds ratio $=156.41,95 \%$ CI [55.72-439.1]) and ICL $(p<0.001$; odds ratio $=77.79,95 \%$ CI [31.55-191.81]) were the strongest predictors of regional lymph node recurrence. Conclusion. We found easily achievable risk factors for RLNR in thyroid cancers patients. We suggested that patients with specific clinicopathological features like male gender, age $\geq 45$ years, larger tumor size, and extrathyroidal extension be considered as prophylactic lymphadenectomy candidates.

\section{Introduction}

Thyroid cancer is the most common cancer of the endocrine system with growing incidence all over the world. Thyroid cancers cause less than $0.5 \%$ of cancer-caused deaths. Most types of thyroid cancer are known to be differentiated with slow clinical progression and cause occasional distant metastasis in patients [1-3]. On the other hand, regional metastasis to cervical lymph nodes is found in about $70 \%$ of thyroid cancers as a part of their clinical development. Regional metastasis to cervical lymph nodes is an independent risk factor of recurrence; although the effect of recurrence on decrement of survival is clearly acceptable, this subject remained a controversy $[4,5]$. It is believed that recurrence plays an important role in clinical manifestation of differentiated thyroid cancers. Previous studies demonstrated different risk factors that influence recurrence of differentiated thyroid cancer: age, tumor size, histological features of the tumor, and regional and distant metastasis [6-8]. Based on a recent study by Wada et al., patients with regional metastasis to cervical lymph nodes have a recurrence rate of $16.3 \%$ while in those without metastasis recurrence rate is zero percent [9]. There are different widespread staging systems to predict prognosis of differentiated thyroid cancers. Nevertheless, some of these systems did not even consider the effect of lymph node involvement and once this effect is included it is based on the anatomical region of lymph nodes and the presence of involvement, not on the basis of histological diagnosis of engagement severity [10-12]. For example, in AJCC scoring system, patients with lymph node metastasis are categorized in Nla and N1b subgroup. In this system, a patient with $2 / 48$ of total cervical lymph nodes is grouped with a patient having $46 / 48$ of metastatic lymph nodes [10]. Considering the fact that the importance and necessity of surgical treatment of lymph nodes is not clearly defined, it is necessary to evaluate the effect of regional lymph node recurrence on 
patients' outcome. In this study, we aimed to investigate this effect and the clinicopathological features indicating prophylactic lymphadenectomy in order to reduce recurrence rate.

\section{Method}

We retrospectively reviewed medical records of 343 patients referred to the general surgery ward of Shariati Hospital, Tehran University of Medical Sciences (TUMS), Tehran, Iran, between March 2007 and March 2013, who underwent thyroidectomy to any extent due to thyroid cancer. Patients managed in other institutions, as well as their pathological specimens, who were referred to our center for second expert opinion were excluded. Required information was collected using the database of the pathology ward of Shariati Hospital and the following data of patients who met the inclusion criteria were gathered: age, gender, anatomical site of tumor, tumor size and weight, presence of single or multinodular goiter, histological type of thyroid cancer, presence of either capsular or vascular or perineural involvement, and regional lymph node metastasis. The ethnic committee of Shariati Hospital approved our study protocol.

All patients with node-positive PTC or other nodepositive thyroid cancers had total thyroidectomy with systematic lymph node dissection of the central neck compartment. The other lymph nodes were dissected as if they were clinically involved. All operations were conducted by a single surgical team in our center.

Thyroid-stimulating hormone suppression therapy was set for all the patients after surgery, and those who were diagnosed with LN metastasis, capsular invasion, or extrathyroidal extension according to pathological reports of thyroid specimen received additional postsurgical radioactive iodine treatment. ${ }^{131}$ I ablative therapy was administered after levothyroxine withdrawal for at least 4 weeks. A whole body scan (WBS) was performed 7 days after ${ }^{131}$ I therapy in order to evaluate whether ablation was successful. Patients received a routine periodic clinical examination every 3 months in the first year and then at 6-month intervals for the following year and then at yearly intervals. Each visit consists of neck ultrasound, WBS, measurement of serum free T4, thyroidstimulating hormone (TSH), thyroglobulin (Tg), and anti-Tg antibodies. tion.

All patients were categorized based on TNM classifica-

The node recurrence was defined as either pathologically approved disease on excision or cytology or recurrent disease confirmed by elevated Tg and whole body scan (WBS) in patients that underwent total thyroidectomy.

All clinicopathological factors potentially known to have an effect on regional recurrence were assessed using univariate analysis using Fisher's exact or chi-squared tests. Significant variables in the univariate analysis were examined in multivariate analysis using a binary logistic regression test. SPSS version 16.0 software (SPSS Inc., Chicago, IL) was used for statistical analysis. Statistical significance was defined as a $p$ value $<0.05$.
TABLE 1: Demographic and clinicopathological characteristics.

\begin{tabular}{lc}
\hline Characteristics & Count $(\%)$ \\
\hline Gender & \\
Female & $245(71.4 \%)$ \\
Male & $98(28.6 \%)$ \\
\hline Disease manifestation & \\
Isolated thyroid nodule & $141(44.31 \%)$ \\
Thyroid nodule + cervical LAP & $64(20.3 \%)$ \\
Isolated cervical LAP & $102(32.3 \%)$ \\
$\quad$ Central cervical LAP & $1(0.98 \%)$ \\
$\quad$ Unilateral cervical LAP & $39(38.235 \%)$ \\
$\quad$ Bilateral cervical LAP & $52(50.98 \%)$ \\
$\quad$ Central + (unilateral or bilateral) cervical LAP & $4(3.922 \%)$ \\
$\quad$ Submandibular LAP & $6(5.882 \%)$ \\
Distant lymph node metastasis & $9(2.8 \%)$ \\
$\quad$ Supraclavicular LAP & $3(0.9 \%)$ \\
$\quad$ Subclavicular LAP & $2(0.6 \%)$ \\
$\quad$ Mediastinal LAP & $4(1.3 \%)$ \\
Skin metastasis & $1(0.316 \%)$ \\
\hline Cervical lymphadenopathy & \\
NA & $120(35.0 \%)$ \\
No cervical LAP & $31(9.0 \%)$ \\
Paratracheal LAP & $5(1.5 \%)$ \\
Pretracheal or central LAP & $25(7.3 \%)$ \\
Unilateral, bilateral, or contralateral cervical LAP & $152(44.3 \%)$ \\
Retropharyngeal or superior mediastinal LAP & $4(1.2 \%)$ \\
Distant lymph node metastasis & $6(1.7 \%)$ \\
\hline &
\end{tabular}

\section{Results}

The clinicopathological history of 343 patients with thyroid cancer was reviewed. The median \pm interquartile range (IQR) of participants' age was $40 \pm 25$ years, and 245 (71.4\%) patients were female. Demographic and clinicopathological findings are represented in Tables 1, 2, and 3. Cancer histopathology of the majority of patients $(86.3 \%)$ was papillary thyroid carcinoma (PTC), and also most of the patients (57.1\%) were in stage I. Also, $76.9 \%$ of the patients had multinodular goiter (MNG). Capsular invasion, vascular invasion, and perineural invasion were found in $60.4 \%, 16.1 \%$, and $21.9 \%$ of the patients, respectively. Moreover, regional lymph node recurrence occurred in approximately half of the patients (49.6\%). Only 12 (3.5\%) of patients showed distant metastasis at their initial presentation.

Cancer pathology was significantly associated with disease stage; so medullary, follicular, and anaplastic types were more associated with advanced stages $(p<0.0001$, Cramer's $V=0.383$ ). Also, patients with follicular thyroid cancer (FTC) were approximately 15 years older than PTC patients $(p=0.018)$. Stage I patients were significantly younger than patients with higher stages $(p<0.0001)$. Male gender was found to be more common among medullary, follicular, and anaplastic types $(p=0.003$; odds ratio $=2.511,95 \%$ CI [1.337-4.717]). Regarding microscopic tumor invasion, vascular invasion was found more commonly in medullary, 
TABLE 2: Demographic and clinicopathological characteristics.

\begin{tabular}{|c|c|}
\hline Characteristics & Count (\%) \\
\hline \multicolumn{2}{|l|}{ Goiter } \\
\hline Single & $36(23.1 \%)$ \\
\hline Multinodular & $120(76.9 \%)$ \\
\hline \multicolumn{2}{|l|}{$\mathrm{T}$} \\
\hline $\mathrm{T} 1$ & $61(17.8 \%)$ \\
\hline $\mathrm{T} 2$ & $22(6.4 \%)$ \\
\hline $\mathrm{T} 3$ & $195(56.9 \%)$ \\
\hline $\mathrm{T} 4 \mathrm{a}$ & $43(12.5 \%)$ \\
\hline $\mathrm{T} 4 \mathrm{~b}$ & $22(6.4 \%)$ \\
\hline \multicolumn{2}{|l|}{$\mathrm{N}$} \\
\hline $\mathrm{Nx}$ & $126(36.7 \%)$ \\
\hline No & $31(9.0 \%)$ \\
\hline Nla & $30(8.7 \%)$ \\
\hline N1b & $156(45.5 \%)$ \\
\hline \multicolumn{2}{|l|}{ M } \\
\hline Mx or M0 & $331(96.5 \%)$ \\
\hline M1 & $12(3.5 \%)$ \\
\hline \multicolumn{2}{|l|}{ Capsular invasion } \\
\hline No & $38(39.6 \%)$ \\
\hline Yes & $58(60.4 \%)$ \\
\hline \multicolumn{2}{|l|}{ Vascular invasion } \\
\hline No & $125(83.9 \%)$ \\
\hline Yes & $24(16.1 \%)$ \\
\hline \multicolumn{2}{|l|}{ Perineural invasion } \\
\hline No & $25(78.1 \%)$ \\
\hline Yes & $7(21.9 \%)$ \\
\hline \multicolumn{2}{|l|}{ Lymph node metastasis (LNM) } \\
\hline No LNM & $65(27.5 \%)$ \\
\hline LNM at initial presentation & $54(22.9 \%)$ \\
\hline Regional lymph node recurrence & $117(49.6 \%)$ \\
\hline
\end{tabular}

follicular, and anaplastic types $(p=0.013$; odds ratio $=$ 4.792, 95\% CI [1.488-15.428]). Also, binary logistic regression showed us that the risk of capsular invasion increased by $2.7 \%$ (95\% CI: 1-5.5\%) along with each one-year increase in the age $(p=0.049)$. Also, the risk of perineural invasion increased by 7.9\% (95\% CI: 1.1-15.2\%) along with each one-year increase in the age $(p=0.022)$. MNG was significantly correlated with female gender $(p=0.034$; odds ratio $=2.384,95 \% \mathrm{CI}$ [1.052-5.402]). Metastasis (M1) was more likely to happen in medullary, follicular, and anaplastic types ( $p=0.003$; odds ratio $=6.902,95 \% \mathrm{CI}[2.125-22.419]$ ). Also, we observed that each one-year increase in age was associated with 5.5\% (95\% CI: $1.8-9.3 \%)$ increase in the risk of metastasis ( $p=0.004)$.

Regarding the risk of regional lymph node recurrence, we observed that male gender, age $\geq 45$ years, non-PTC (i.e., medullary, follicular, and anaplastic types) histopathology, T3 (i.e., tumor more than $4 \mathrm{~cm}$ in the greatest dimension limited to the thyroid or any tumor with minimal extrathyroid extension such as extension to sternothyroid muscle or perithyroid soft tissues), stage IVa, and isolated cervical LAP as initial disease manifestation are significant risk factors.
Among these risk factors, T3 $(p<0.001$; odds ratio $=156.41$, 95\% CI [55.72-439.1]) and isolated cervical LAP at initial presentation $(p<0.001$; odds ratio $=77.79$, 95\% CI $[31.55-$ 191.81]) were the strongest predictors of regional lymph node recurrence.

\section{Discussion}

Although the overall prognosis of PTC is good, local recurrence after initial surgery stays an issue; also, in other thyroid cancers, clinicopathological factors predicting local recurrences are unclear [13]. In this regard, performing prophylactic lymph node dissection is a controversy $[14,15]$. Some authors advised lymph node dissection because of a reduction in postoperative thyroglobulin $(\mathrm{Tg})$ levels and also a decrease in morbidity rate which is obtained in the first operation [16-18] and other investigators suggested that this procedure increases the risk of injury to local tissues like parathyroid glands and recurrent laryngeal nerves without any significant effect on survival [19-22]. Thus, clinicopathological predictors of greater recurrence risk in lymph node would be valuable.

In this study, we found that male gender, age $\geq 45$ years, non-PTC histopathology, and T3 stage are significant risk factors of regional lymph node recurrence. Among these risk factors, T3 stage $(p<0.001$; odds ratio $=156.41$, 95\% CI [55.72-439.1]) and isolated cervical LAP at initial presentation $(p<0.001$; odds ratio $=77.79$, 95\% CI $[31.55-$ 191.81]) were the strongest predictors of regional lymph node recurrence.

Previous studies proposed that male gender, large tumor size, extrathyroidal extension, capsular invasion, and lymphovascular invasion are predictors of both prognosis in thyroid cancers [23] and risk factors for central lymph node metastasis [24]. These findings are comparable to ours.

$\mathrm{Qu}$ et al. showed that, in a group of 514 male and 2128 female patients, $26.3 \%$ of the patients with regional lymph node recurrence were male against $15 \%$ of the patients without [24].

Studies investigating the effect of age in RLNR in patients with PTC suggested that age $<20$ years and age $>70$ years have a poor prognosis independent of cancer stage $[25,26]$. In our study, the incidence of regional lymph node recurrence was higher in patients aged $\geq 45$ years.

It is believed that large tumor size at presentation is associated with higher risk of regional recurrence $[27,28]$. Tumor size greater than $0.5 \mathrm{~cm}$ in diameter has been proposed to strongly be associated with regional lymph node recurrence $[29,30]$. We reported that tumor size $>4 \mathrm{~cm}$ in the greatest dimension is more likely to precede regional lymph node recurrence.

The effect of extrathyroidal extension has been investigated in some studies and the elevated risk of regional lymph node recurrence has been reported as a result of capsular invasion, vascular invasion, and perineural invasion [31, 32]. Scheumann and colleagues reported that extrathyroid extension or higher $\mathrm{T}$ stage is associated with higher rate of local recurrence and lower survival rate, as well as an increase 
TABLE 3: Demographic and clinicopathological characteristics.

\begin{tabular}{lc}
\hline Characteristics & Count (\%) \\
\hline Cancer type & $289(86.3 \%)$ \\
Papillary thyroid carcinoma (PTC) & $26(7.8 \%)$ \\
Medullary thyroid carcinoma (MTC) & $16(4.8 \%)$ \\
Follicular thyroid carcinoma (FTC) or Hürthle cell carcinoma (HCC) & $4(1.2 \%)$ \\
Anaplastic (undifferentiated) thyroid carcinoma (ATC or UTC) & $192(57.1 \%)$ \\
Stage & $15(4.5 \%)$ \\
I & $29(8.6 \%)$ \\
II & $81(24.1 \%)$ \\
III & $11(3.3 \%)$ \\
IVa & $8(2.4 \%)$ \\
IVb & \\
IVc & \\
Capsule status & $31(24.4 \%)$ \\
Uncapsulated & $26(20.5 \%)$ \\
Capsulated & \\
Partially capsulated & \\
\hline
\end{tabular}

in lymph node recurrence [33]. We showed that patients with T3 stage based on TNM classification are at greater risks for developing regional lymph node recurrence.

Baek et al., based on multivariate analysis results, stated that only the $\mathrm{N}$ stage was significantly related to regional recurrence, and the disease-free survival period was significantly shorter in the lymph node metastasis-positive group than in the lymph node metastasis-negative group [34]. We found cervical LAP at initial presentation, one of the strongest predictors of regional lymph node recurrence, to be a significant risk factor.

The strongest predictor of regional lymph node recurrence was tumor size more than $4 \mathrm{~cm}$ and extrathyroid extension. Tumor cells generally spread through the lymphatic system from the thyroid gland to the central and lateral compartments [35]. Skip metastasis to the lateral compartment of the neck in the absence of central disease is uncommon [35].

This study has limitations. Our study was performed retrospectively and not all patients had the same follow-up period. Also, our criteria for diagnosis of regional lymph node recurrence were not completely capable of finding each recurrence so we might have missed some cases.

\section{Conclusion}

Our study revealed several risk factors for secondary LNM in patients with thyroid cancers, all of which are easily achievable in clinical settings. The identification of patients with greater risks of secondary LNM has a great impact on their survival. In this regard, a group of patients who undergo prophylactic lymphadenectomy ultimately have better outcomes. We suggested that patients with specific clinicopathologic features like male gender, larger tumor size, and extrathyroidal extension be considered as candidates for prophylactic lymphadenectomy.

\section{Competing Interests}

The authors declare that they have no competing interests.

\section{References}

[1] K. M. Kim, J. B. Park, K. S. Bae, C. B. Kim, D. R. Kang, and S. J. Kang, "Clinical prognostic index for recurrence of papillary thyroid carcinoma including intraoperative findings," Endocrine Journal, vol. 60, no. 3, pp. 291-297, 2013.

[2] M. J. Schlumberger, "Papillary and follicular thyroid carcinoma," The New England Journal of Medicine, vol. 338, no. 5, pp. 297-306, 1998.

[3] E. L. Mazzaferri, "Papillary thyroid carcinoma: factors influencing prognosis and current therapy," Seminars in Oncology, vol. 14 , no. 3, pp. 315-332, 1987.

[4] J. Ricarte-Filho, I. Ganly, M. Rivera et al., "Papillary thyroid carcinomas with cervical lymph node metastases can be stratified into clinically relevant prognostic categories using oncogenic BRAF, the number of nodal metastases, and extranodal extension," Thyroid, vol. 22, no. 6, pp. 575-584, 2012.

[5] G. Teixeira, T. Teixeira, F. Gubert, H. Chikota, and R. Tufano, "The incidence of central neck micrometastatic disease in patients with papillary thyroid cancer staged preoperatively and intraoperatively as N0," Surgery, vol. 150, no. 6, pp. 1161-1167, 2011.

[6] A. Jukkola, R. Bloigu, T. Ebeling, P. Salmela, and G. Blanco, "Prognostic factors in differentiated thyroid carcinomas and their implications for current staging classifications," EndocrineRelated Cancer, vol. 11, no. 3, pp. 571-579, 2004.

[7] A. R. Shaha, "Implications of prognostic factors and risk groups in the management of differentiated thyroid cancer," Laryngoscope, vol. 114, no. 3, pp. 393-402, 2004. 
[8] S. L. Cushing, C. E. Palme, N. Audet, S. Eski, P. G. Walfish, and J. L. Freeman, "Prognostic factors in well-differentiated thyroid carcinoma," Laryngoscope, vol. 114, no. 12, pp. 2110-2115, 2004.

[9] N. Wada, N. Suganuma, H. Nakayama et al., "Microscopic regional lymph node status in papillary thyroid carcinoma with and without lymphadenopathy and its relation to outcomes," Langenbeck's Archives of Surgery, vol. 392, no. 4, pp. 417-422, 2007.

[10] S. B. Edge and C. C. Compton, "The american joint committee on cancer: the 7th edition of the AJCC cancer staging manual and the future of TNM," Annals of Surgical Oncology, vol. 17, no. 6, pp. 1471-1474, 2010.

[11] I. D. Hay, C. S. Grant, W. F. Taylor, and W. M. McConahey, "Ipsilateral lobectomy versus bilateral lobar resection in papillary thyroid carcinoma: a retrospective analysis of surgical outcome using a novel prognostic scoring system," Surgery, vol. 102, no. 6, pp. 1088-1095, 1987.

[12] S. I. Sherman, J. D. Brierley, M. Sperling et al., "Prospective multicenter study of thyroid carcinoma treatment: initial analysis of staging and outcome," Cancer, vol. 83, no. 5, pp. 1012-1021, 1998.

[13] J. Lee, Y. Song, and E. Y. Soh, "Central lymph node metastasis is an important prognostic factor in patients with papillary thyroid microcarcinoma," Journal of Korean Medical Science, vol. 29, no. 1, pp. 48-52, 2014.

[14] B. H.-H. Lang, S.-H. Ng, L. L. H. Lau, B. J. Cowling, K. P. Wong, and K. Y. Wan, "A systematic review and metaanalysis of prophylactic central neck dissection on short-term locoregional recurrence in papillary thyroid carcinoma after total thyroidectomy," Thyroid, vol. 23, no. 9, pp. 1087-1098, 2013.

[15] J. A. Sosa, "Is routine prophylactic central neck dissection indicated for low-risk papillary thyroid cancer: can we determine cost-effectiveness if we are unsure about its effectiveness and safety?" Surgery, vol. 154, no. 6, pp. 1146-1147, 2013.

[16] I. J. Nixon, I. Ganly, S. G. Patel et al., "Observation of clinically negative central compartment lymph nodes in papillary thyroid carcinoma," Surgery, vol. 154, no. 6, pp. 1166-1173, 2013.

[17] M. Raffaelli, C. De Crea, L. Sessa et al., "Prospective evaluation of total thyroidectomy versus ipsilateral versus bilateral central neck dissection in patients with clinically node-negative papillary thyroid carcinoma," Surgery, vol. 152, no. 6, pp. 957-964, 2012.

[18] B. M. Sadowski, S. K. Snyder, and T. C. Lairmore, "Routine bilateral central lymph node clearance for papillary thyroid cancer," Surgery, vol. 146, no. 4, pp. 696-705, 2009.

[19] D. Giordano, R. Valcavi, G. B. Thompson et al., "Complications of central neck dissection in patients with papillary thyroid carcinoma: results of a study on 1087 patients and review of the literature," Thyroid, vol. 22, no. 9, pp. 911-917, 2012.

[20] N. Palestini, A. Borasi, L. Cestino, M. Freddi, C. Odasso, and A. Robecchi, "Is central neck dissection a safe procedure in the treatment of papillary thyroid cancer? Our experience," Langenbeck's Archives of Surgery, vol. 393, no. 5, pp. 693-698, 2008.

[21] J.-L. Roh, J.-M. Kim, and C. I. Park, "Central cervical nodal metastasis from papillary thyroid microcarcinoma: pattern and factors predictive of nodal metastasis," Annals of Surgical Oncology, vol. 15, no. 9, pp. 2482-2486, 2008.

[22] D. Viola, G. Materazzi, L. Valerio et al., "Prophylactic central compartment lymph node dissection in papillary thyroid carcinoma: clinical implications derived from the first prospective randomized controlled single institution study," Journal of
Clinical Endocrinology and Metabolism, vol. 100, no. 4, pp. 13161324, 2015.

[23] D. S. Cooper, G. M. Doherty, B. R. Haugen et al., "Revised American thyroid association management guidelines for patients with thyroid nodules and differentiated thyroid cancer," Thyroid, vol. 19, no. 11, pp. 1167-1214, 2009.

[24] H. Qu, G.-R. Sun, Y. Liu, and Q.-S. He, "Clinical risk factors for central lymph node metastasis in papillary thyroid carcinoma: a systematic review and meta-analysis," Clinical Endocrinology, vol. 83, no. 1, pp. 124-132, 2015.

[25] L. Vini, S. L. Hyer, J. Marshall, R. A’Hern, and C. Harmer, “Longterm results in elderly patients with differentiated thyroid carcinoma," Cancer, vol. 97, no. 11, pp. 2736-2742, 2003.

[26] D. Landau, L. Vini, R. A’Hern, and C. Harmer, “Thyroid cancer in children: the Royal Marsden Hospital experience," European Journal of Cancer, vol. 36, no. 2, pp. 214-220, 2000.

[27] A. R. Shaha, J. P. Shah, and T. R. Loree, "Risk group stratification and prognostic factors in papillary carcinoma of thyroid," Annals of Surgical Oncology, vol. 3, no. 6, pp. 534-538, 1996.

[28] A. R. Shaha, J. P. Shah, and T. R. Loree, "Low-risk differentiated thyroid cancer: the need for selective treatment," Annals of Surgical Oncology, vol. 4, no. 4, pp. 328-333, 1997.

[29] K.-E. Kim, E.-K. Kim, J. H. Yoon, K. H. Han, H. J. Moon, and J. Y. Kwak, "Preoperative prediction of central lymph node metastasis in thyroid papillary microcarcinoma using clinicopathologic and sonographic features," World Journal of Surgery, vol. 37, no. 2, pp. 385-391, 2013.

[30] Q. Zhao, J. Ming, C. Liu et al., "Multifocality and total tumor diameter predict central neck lymph node metastases in papillary thyroid microcarcinoma," Annals of Surgical Oncology, vol. 20, no. 3, pp. 746-752, 2013.

[31] S. Miao, X. Mao, R. Pei et al., "Predictive factors for different subgroups of central lymph node metastasis in unilateral papillary thyroid carcinoma," Journal for Oto-Rhino-Laryngology and Its Related Specialties, vol. 75, no. 5, pp. 265-273, 2013.

[32] S. M. Jara, K. A. Carson, S. I. Pai et al., "The relationship between chronic lymphocytic thyroiditis and central neck lymph node metastasis in North American patients with papillary thyroid carcinoma," Surgery, vol. 154, no. 6, pp. 1272-1282, 2013.

[33] G. F. W. Scheumann, O. Gimm, G. Wegener, H. Hundeshagen, and $\mathrm{H}$. Dralle, "Prognostic significance and surgical management of locoregional lymph node metastases in papillary thyroid cancer," World Journal of Surgery, vol. 18, no. 4, pp. 559$568,1994$.

[34] S.-K. Baek, K.-Y. Jung, S.-M. Kang et al., "Clinical risk factors associated with cervical lymph node recurrence in papillary thyroid carcinoma," Thyroid, vol. 20, no. 2, pp. 147-152, 2010.

[35] J. S. Jeong, H. K. Kim, C.-R. Lee et al., "Coexistence of chronic lymphocytic thyroiditis with papillary thyroid carcinoma: clinical manifestation and prognostic outcome," Journal of Korean Medical Science, vol. 27, no. 8, pp. 883-889, 2012. 


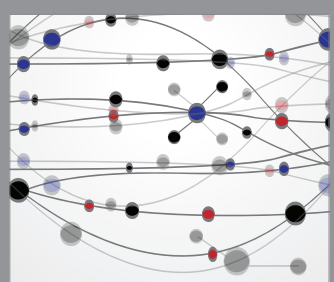

The Scientific World Journal
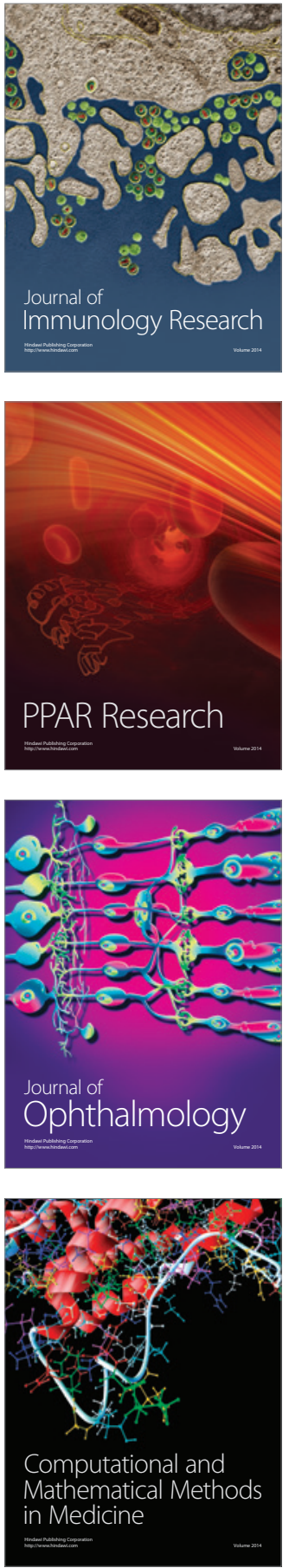

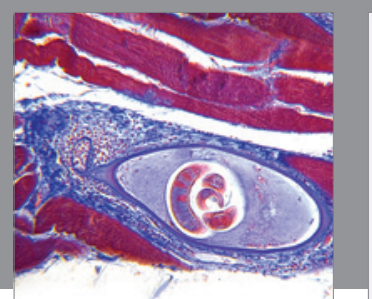

Gastroenterology Research and Practice

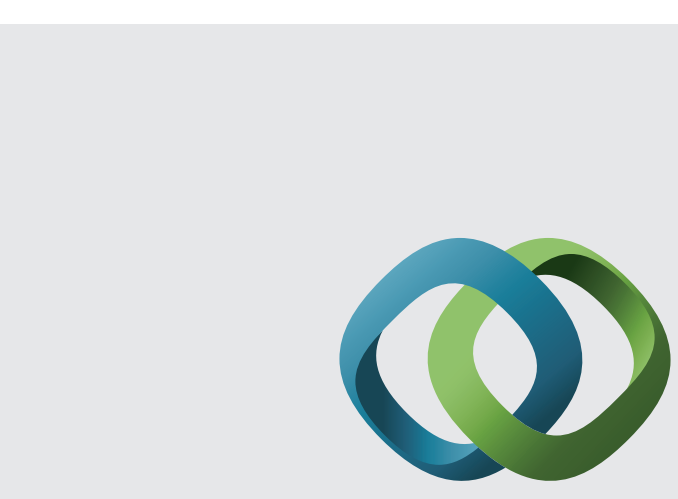

\section{Hindawi}

Submit your manuscripts at

http://www.hindawi.com
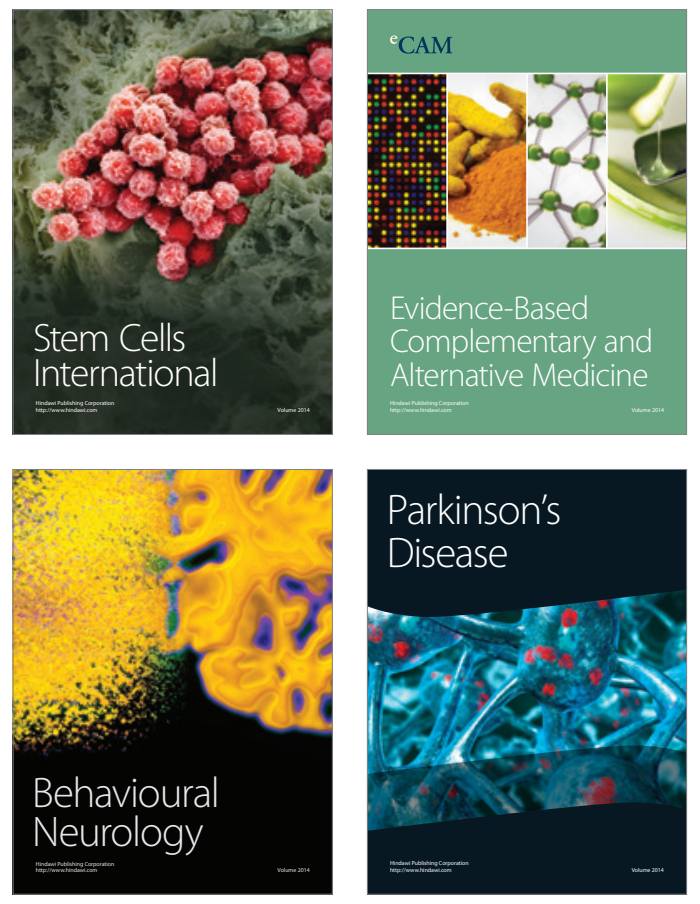
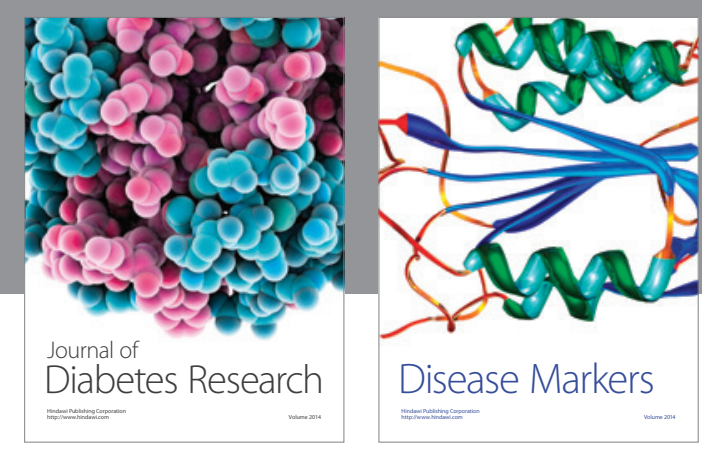

Disease Markers
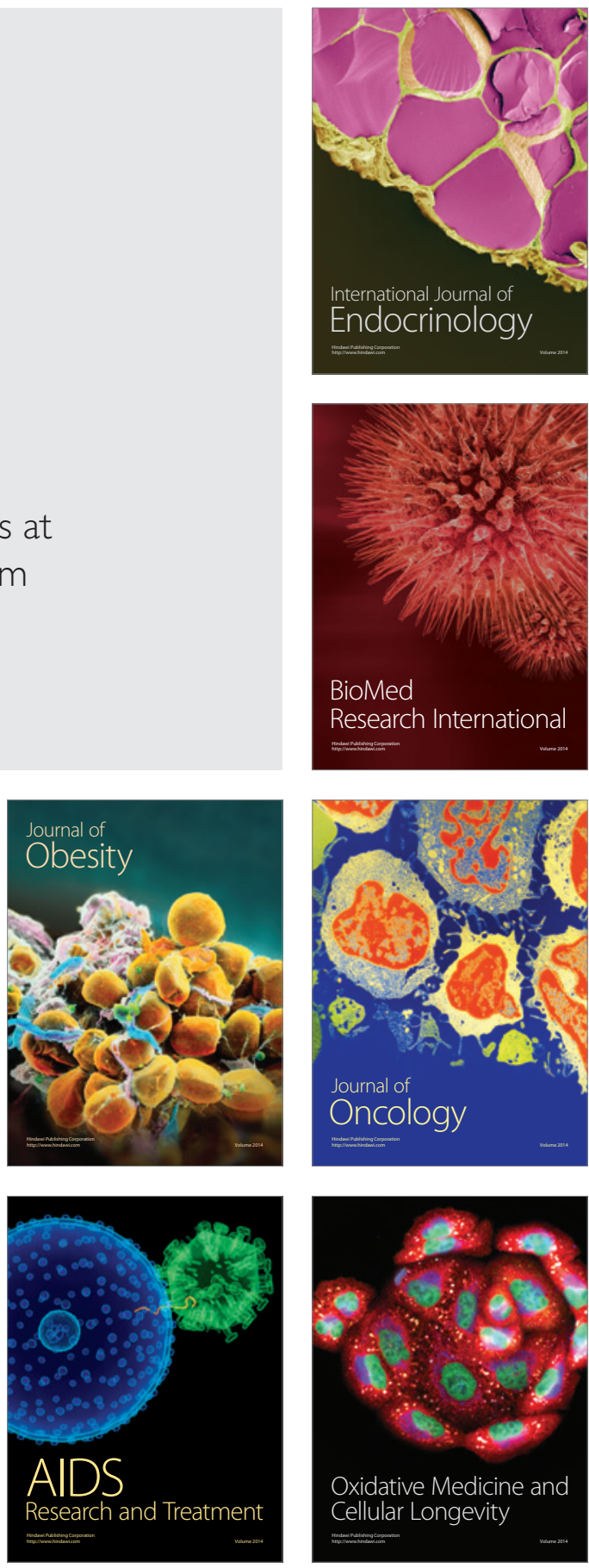